\title{
Configurational entropy of microemulsions: The fundamental length scale
}

\author{
Howard Reiss, H. Michael Ellerby, and Jose A. Manzanares ${ }^{a)}$ \\ Department of Chemistry and Biochemistry, University of California, Los Angeles, Los Angeles, \\ California 90024-1569
}

(Received 11 May 1993; accepted 7 September 1993)

\begin{abstract}
Phenomenological models have been quite successful in characterizing both the various complex phases and the corresponding phase diagrams of microemulsions. In some approaches, e.g., the random mixing model (RMM), the lattice parameter is of the order of the dimension of an oil or water domain and has been used as a length scale for computing a configurational entropy, the so-called entropy of mixing, of the microemulsion. In the central and material section of this paper (Sec. III), we show that the fundamental length scale for the calculation of the entropy of mixing is of the order of the cube root of the volume per molecule-orders of magnitude smaller than the dimension of such a domain. This length scale is specifically the scale for the configurational entropy-not that which measures either the curvature of the interface, the "granularity" of the microemulsion, or the persistence length. Furthermore, we demonstrate, in general, that mixing entropy, evaluated in configuration space as opposed to phase space, will not be physically correct unless it is made to be consistent with the phase space evaluation. Following this core section, we give a one-dimensional illustration of the problem (Sec. IV), and discuss the consequences of our general result with respect to the RMM (Sec. V). The RMM not only seriously underestimates the entropy of mixing but exhibits a dependence on composition that is qualitatively very different from the correct dependence. Furthermore, for oil or water rich compositions of the microemulsion, the correct mixing entropy reinforces effects that would normally be attributed to bending energy, i.e., it destabilizes the system.
\end{abstract}

\section{INTRODUCTION}

During the past decade, considerable progress has been made in the understanding of microemulsions. An important approach underlying much of this progress has been the use of phenomenological lattice methods, or more broadly, a phenomenological model in the description of the microemulsion. This model views the microemulsion phase as either a dispersion of oil in water (Winsor I), a dispersion of water in oil (Winsor II), or a bicontinuous mixture of oil and water phases (Winsor III). ${ }^{1}$ In all cases the oil and water are mesophases, having dimensions on an intermediate scale, e.g., $50 \mathrm{~nm}$. On the macroscopic level the microemulsion behaves as a stable phase subject to the ordinary laws of thermodynamics. Figure 1 provides a schematic view of this system in which the shaded regions represent domains of oil, while the unshaded region represents water. In the actual system there are surfactant, and frequently cosurfactant, molecules localized to the extended interface between the two mesophases. These reduce the interfacial tension to a vanishingly small value.

Some aspects of this approach go back to Reiss, ${ }^{2}$ and Talmon and Prager, ${ }^{3}$ although none of these authors employed a lattice. Reiss performed his analysis in the continuum, while Talmon and Prager utilized a system of Voronoi polyhedra.

Phenomenological lattice models have been investigated by Ruckenstein, ${ }^{4}$ De Gennes and Taupin, ${ }^{5}$ Jouffroy, Levinson, and De Gennes, ${ }^{6}$ Widom, ${ }^{7}$ and Andelman et al. ${ }^{8}$

a)Permanent address: Departamento de Termodimámica, Facultad de Física, Universidad de Valencia, E-46100 Burjasot, Spain.
These models employ a lattice parameter $\xi$ as a length scale to calculate the configurational (mixing) entropy of the microemulsion. The lattice parameter is typically of the order of the dimension of an oil or water mesophase. However, in the core section (Sec. III) of this paper, we show that the fundamental length scale for the calculation of the entropy of mixing is of the order of the cube root of the volume per molecule-orders of magnitude smaller than the dimension of a mesophase domain. Following this central theme, we give a simple illustration of the length scale calculation, and discuss the consequences of our result with respect to phenomenological models of microemulsions, specifically the random mixing model (RMM).$^{5-8}$

\section{THE PHENOMENOLOGICAL MODEL}

In the phenomenological model, the Gibbs free energy of the microemulsion is approximated by the following expression:?

$$
G=N_{d} \mu_{o}+N_{u} \mu_{w}+\gamma \Sigma-T S_{\text {mix }},
$$

where $N_{o}$ and $N_{w}$ are the total numbers of oil and water molecules in the system, $\mu_{o}$ and $\mu_{w}$ are the chemical potentials of oil and water in the corresponding bulk phases, $\gamma$ is the interfacial tension between the mesophases, $\Sigma$ is the area of the extended interface, and $T$ is the temperature. Thus, the first two terms on the right-hand side of Eq. (1) represent the free energy of the oil and water mesophases, respectively, while the third term represents the surface free energy. The rationale for the first three terms on the right-hand side of Eq. (1) is that, to a high degree of approximation, the mesophases are large enough to possess intensive properties identical to those of the bulk phases. 


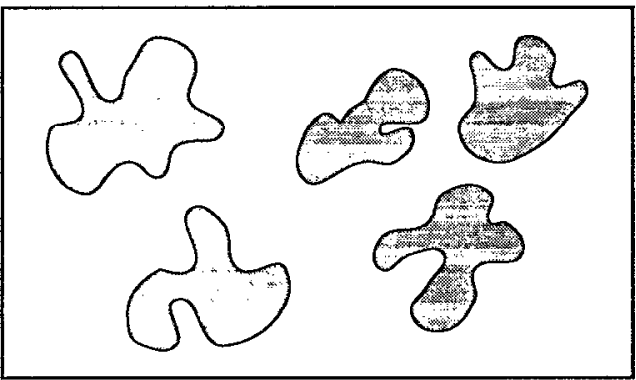

FIG. 1. Schematic representation of a region within a microemulsion (Winsor I). Shaded regions are oil domains. Unshaded region represents water.

As is conventional, the effects of bending energy are included in the value of $\gamma$. However, this does not imply that bending energy effects are merely included in a multiplicative factor $\gamma$ that is itself independent of $\Sigma$. As indicated below, bending energy effects also appear in $S_{\text {mix }}$, since the allowable configurations of the system are affected by bending energy.

The last term on the right-hand side of Eq. (1) is something new, and its inclusion represents a subtle combination of microscopic and macroscopic ideas. $S_{\operatorname{mix}}$ is a configurational entropy, in this case the so-called entropy of mixing, which is supposed to correspond to the enormous number of patterns $\Omega$, that can be generated by translating and deforming the domains of oil and water through all possible configurations that conserve the respective volumes of oil and water, as well as the total interfacial area $\Sigma$. Bending energy may also affect the allowable configurations so that its effects appear in both the next to the last, and the last term of Eq. (1). Different patterns are generated by the continuous movement of the surfaces that represent the interface so that an infinite value for $\Omega$ would result. Clearly then, not all such patterns contribute to $\Omega$. Before a new pattern can be considered to have been generated an element of interface must be displaced some minimum distance defined by a length scale $\lambda$. Thus the entropy of mixing is defined as

$$
S_{\operatorname{mix}}(\lambda)=k \ln \Omega(\lambda)
$$

where $k$ is Boltzmann's constant. Assuming that $\mu_{o}, \mu_{w}, \gamma$, and $\Sigma$ are known, $S_{\operatorname{mix}}$ is essentially the residual entropy after the first three terms on the right-hand side of Eq. (1) have been accounted for.

This characterization of $S_{\mathrm{mix}}$ is not sufficient for specifying either its functional form or the magnitude of $\lambda$. As we emphasize later, fundamentals require that physical entropy be evaluated through the counting of quantum states in phase space, and the contribution of configuration space to this process cannot be separated in an absolute manner. As a result, that part of the entropy which is configurational must be defined, and this is usually done within the context of some model. Although the intent of the phenomenological model with respect to the definition of con- figurational entropy is fairly clear, a precise definition does not seem to have been advanced. Therefore, it is useful to sharpen this intent into a clear definition.

For this purpose it is convenient to begin with consideration of the random mixing model, ${ }^{5-8}$ a particular example of the phenomenological model. The RMM introduces a course-grained lattice of cells in which each cell is filled with continuous fluid, all oil or all water. If the volume fractions of oil and water are denoted by $\varphi$ and $(1-\varphi)$, respectively, then $\Omega$ for the entropy of mixing is obtained by counting the random permutations of oil and water cells over the lattice.

The lattice parameter $\xi$ has been chosen in a number of ways. It has been set equal to the so-called persistence length, ${ }^{5,6}$ or obtained through a variational method, ${ }^{7}$ or determined so that upon "random mixing" the interfacial area would be exactly equal to area required to accommodate a saturated layer of all the available surfactant. ${ }^{8}$ The lattice parameter $\xi$ serves as a scale for the dispersion, and its value is typically of the order of the dimension of the oil and water mesophases, e.g., $50 \mathrm{~nm}$-much larger than the size of a molecule. If the third method (above) for determining $\xi$ is used, one finds ${ }^{8,10}$

$$
\xi=\frac{z v^{1 / 3} \varphi(1-\varphi)}{\left(N_{\text {surf }} / N\right)}
$$

where $N$ is the total number of molecules (oil and water) in the system, $v$ is the average volume per molecule in the system, $z$ is the lattice coordination number, and $N_{\text {surf }}$ is the number of surfactant molecules assumed to occupy an interfacial area $N_{\text {surf }} t^{2 / 3}$. An obvious modification of the RMM would involve the abandonment of the random permutations of the oil and water cells in favor of allowing only those permutations that conserve $\Sigma$, whatever the value of $\xi$.

In the continuum version of the phenomenological model, as we have indicated, we are faced with a continuum of patterns that amounts to an infinite value for $\Omega$ which must then be rationalized by the introduction of the length scale. But the use of the length scale is really equivalent to the use of the phenomenological lattice, as in the case of the RMM, with the difference that $\lambda$ is not determined, as in the RMM, by Eq. (3), i.e., oil and water cells are no longer mixed randomly! Thus $\lambda$ remains a lattice parameter.

If the correct value of $\lambda$ proves to be small enough, the precise symmetry of the lattice will not be crucial to its determination or to the evaluation of $S_{\text {mix }}(\lambda)$. There will be a small latitude in the specification of the interfacial area $\Sigma$. For example, one might choose for $\Sigma$, as in the case of the RMM, the sum of the cell faces that have oil on one side and water on the other. If the lattice parameter was of the size of a molecule this would lead to an interface that was irregular on the molecular scale, and therefore not very representative of the usual transition zone between two coexisting liquid phases. A smooth surface traced through the neighborhood of this irregular surface would be preferable, and irregularities would then become part of the interfacial entropy. In any event the extents of the 
irregular and the smooth surfaces would differ at most by a factor of the order of the coordination number of the lattice.

We are now in a position to provide a firm definition for $S_{\text {mix }}(\lambda)$ in the phenomenological model. $S_{\text {mix }}(\lambda)$ is $k \ln \Omega(\lambda)$, where $\Omega(\lambda)$ is the number of permutations of oil and water cells on a phenomenological lattice of lattice parameter $\lambda$ where the permutations are restricted to those that conserve the interfacial area required to accommodate a saturated layer of surfactant. If the requirement of saturation is relaxed or replaced, or others are added to it, then $\Omega(\lambda)$ is the number of permutations allowed by whatever alternative set of constraints (if any) are applied. It should be noted that the continuum limit is included in this definition by setting $\lambda=0$.

It is still necessary to introduce the condition that determines $\lambda$. The possibility of introducing this condition is a consequence of the degree of freedom released by abandoning Eq. (3), and the obvious way to use it is to choose a condition that insures that $S_{\text {mix }}(\lambda)$ is physically consistent with the fundamental requirement that entropy be evaluated through the counting of quantum states in phase space. One way to do this would be to require that $S_{\text {mix }}(\lambda)$ satisfy Eq. (1), given that the exact Gibbs free energy $G$ is known. While the exact $G$ is not known for a real microemulsion, it is known for simple models of microemulsions so that some general idea of the proper length scale may be obtained. Furthermore, as we demonstrate below, a fairly good estimate can be derived from the consideration of real systems that are not exactly solvable.

If we denote by $G_{\text {exact }}$, the exact value of the Gibbs free energy, then, using Eq. (1), the condition that $S_{\text {mix }}(\lambda)$ must satisfy in order to be physically consistent can be expressed as

$$
S_{\text {mix }}(\lambda)=\left(N_{o} \mu_{o}+N_{u} \mu_{w}+\gamma \Sigma-G_{\text {exact }}\right) / T .
$$

Before leaving this section, we emphasize that the following section contains the core of our argument, and that the later sections follow as a consequence of the result.

\section{AN ESTIMATE OF THE LENGTH SCALE $\lambda$}

The functional form of $S_{\text {mix }}(\lambda)$ and the length scale $\lambda$ cannot be specified by Eq. (4). However, we can make further progress in this regard by considering the schematic illustration of a microemulsion (here, Winsor $\mathrm{I}$ ) given in Fig. 2. The figure is a "snapshot" of the microemulsion that presents one of the possible configurations of the oil and water domains. Specifically, the figure shows two oil globules, whose molecules are represented by filled circles, surrounded by water, whose molecules are represented by empty circles. The interface between the oil and water domains is delineated by shaded lines.

We can refine this picture of the interface by referring to Fig. 3, which is a highly magnified view of a tiny piece of interface. Here, we have sketched "boundaries" about the molecules, such that a close-packed array of irregular cells is produced, each cell containing one, and only one molecule. This array of imaginary cells is drawn purely for

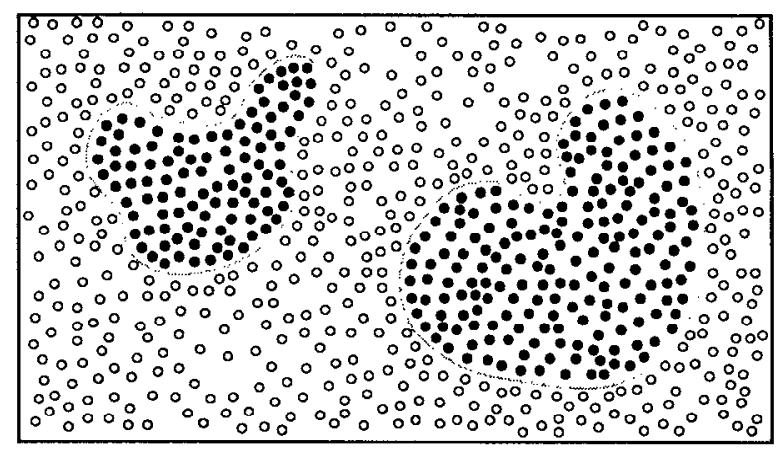

FIG. 2. A microscopic view of a region within a microemulsion (Winsor I). Two oil globules, whose molecules are represented by filled circles, lie in the water domain, whose molecules are represented by empty circles. The oil-water interface is represented by the shaded lines.

visualization, and is not physical-we are dealing with a fluid. Indeed, there is no unique way to construct the array!

The interface itself might be defined as the set of cell faces that separate imaginary cells of oil from imaginary cells of water, shown as the dark line in Fig. 3. Because the construction of the array is itself nonunique, this interface will also not be unique. On the other hand, it is clear that in the thermodynamic limit the possible variation of the area of this interface, allowed by the nonuniqueness, will be small. Thus, we can talk meaningfully in this picture about a constant interface area $\Sigma$, defined by the adjacent oilwater imaginary cell faces. The actual interface between phases in a physical system is of course not a mathematical surface but a transition zone that may be several molecular diameters in thickness. Therefore, it may include more than one cell on either side of the mathematical interface shown in Fig. 3. This will have little effect on the general argument presented below, and any energetic and entropic contributions due to this finite thickness will appear in the $\gamma \Sigma$ term on the right-hand side of Eq. (1).

Now, suppose we fix the interfacial boundaries between the oil and water domains shown in Fig. 2 at a given

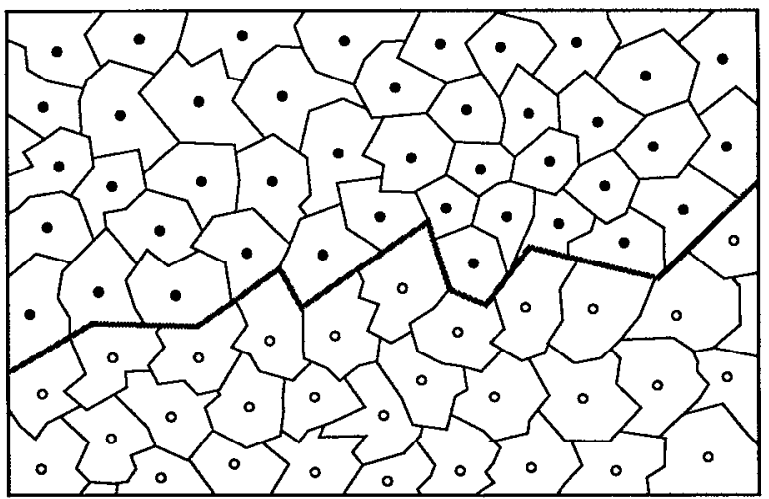

FIG. 3. A highly magnified microscopic view of a tiny piece of the oilwater interface. The filled and empty circles represent oil and water molecules, respectively. The array of irregular cells, each containing a single molecule, is an abstraction useful for defining the interface between oil and water domains. The array is not unique. 
interfacial area $\Sigma$ and given bending energy, and then allow the oil and water molecules to move freely within their respective domains. It is clear from Fig. 2 that, except at the interface, oil molecules are surrounded by oil molecules, and water molecules by water molecules. Thus, if we ignore the interaction between molecules at the interface, the oil molecules are completely decoupled from the water molecules. The partition function $Q^{*}$ for this decoupled, two-component, system of oil and water molecules is then given by the product,

$$
Q^{*}=Q_{o}\left(N_{o}, V_{o}, T\right) Q_{w}\left(N_{w}, V_{w}, T\right),
$$

of the partition function $Q_{o}$ for the oil molecules in their multiply connected domain, times the partition function $Q_{w}$ for the water molecules in their domain, where $V_{o}$ and $V_{w}$ are the corresponding volumes of oil and water. It is important to understand that the configuration integrals corresponding to $Q_{o}$ and $Q_{w}$ "count" configurations of the molecules within their respective domains, but do not "count" the possible configurations of those domains, since the domain boundaries are fixed. It is important to emphasize that in spite of the above appeal to imaginary cells as an expository tool, the problem of the absence of communal entropy ${ }^{11}$ does not arise, since the molecules are not confined to individual cells, but are free to move through the multiply connected domain.

As it stands, $Q^{*}$ of Eq. (5) is also the partition function for one particular configuration of a microemulsion with a given interfacial area and bending energy (again, ignoring interfacial effects). $Q^{*}$ will have the same value for all possible configurations of the oil and water domains, provided that each and every such configuration has the same constant values of the interfacial area $\Sigma$, and bending energy. Furthermore, since $\Sigma$ is constant, the energetic correction due to those molecules at the interface will be constant in all allowable configurations of the domains. As a result, all such allowed configurations will have the same energy.

The total partition function $Q$ of the microemulsion must include all possible configurations $\mathscr{P}$ of the oil and water domains, constrained by a constant interfacial area $\Sigma$, and a constant bending energy. Thus, not only $\gamma$, but $\mathscr{P}$, is affected by bending effects. Although for simplicity of notation we will write $\mathscr{P}$ as a function of $N_{o}, N_{w}$, and $\Sigma$, when additional constraints such as those due to bending are involved, $\mathscr{P}$ must also be a function of the thermodynamic variable (variables) corresponding to the new constraint (constraints).

The number of possible configurations $\mathscr{P}$ of the oil and water domains is just the number of permutations of the oil and water molecules, among the frozen arrangement of the positions of the molecules within the snapshot (Fig. 3), subject to the above constraints, that lead to new patterns of the mesophase domains, or, in other words, new interfacial boundaries as represented by the gray lines of Fig. 3 . It is to be emphasized that we are permuting molecules and not imaginary cells, so that we are indeed generating and counting quantum states to complete the specification of the true physical partition function.
Another equivalent way of looking at the situation involves thinking about the molecules in Figs. 2 and 3 as bearing oil and water "labels." Then, in order to evaluate $\mathscr{P}$, we can think of permuting the labels among the frozen positions, subject to the constraints discussed above.

Without compromising our estimate of the length scale we can, for simplicity, assume that the densities of oil and water are identical, or, in other words,

$$
\rho_{o}=N_{o} / V_{o}=\rho_{w}=N_{w} / V_{w}=\rho,
$$

so that the average volume $\langle v\rangle$ of an imaginary cell is just the average volume per molecule $\rho^{-1}$. The partition function of the microemulsion $Q$ then follows immediately as

$$
\begin{aligned}
& Q\left(N_{o}, N_{w}, \Sigma, V, T\right) \\
& \quad=\mathscr{P}\left(N_{o}, N_{w}, \Sigma\right) Q_{o}\left(N_{o}, V_{o}, T\right) Q_{w}\left(N_{w}, V_{w}, T\right),
\end{aligned}
$$

where $V=V_{o}+V_{w}$, and where it is understood that $\mathscr{P}\left(N_{o}, N_{w}, \Sigma\right)$ is the number of permutations allowed under the constraints discussed above.

Using Eq. (7), we can derive an estimate for $G_{\text {exact }}$, appearing in Eq. (4). Standard relations between $G$ and $Q$ in the canonical ensemble allow us to write

$$
G_{\text {estimate }}=-k T \ln Q+p V,
$$

which yields, upon substitution of Eq. (7),

$$
\begin{aligned}
G_{\text {estimate }}= & -k T \ln \mathscr{P}+\left(-k T \ln Q_{o}+p V_{o}\right) \\
& +\left(-k T \ln Q_{w}+p V_{w}\right) \\
= & -k T \ln \mathscr{P}+G_{o}\left(N_{o}, p, T\right)+G_{w}\left(N_{w}, p, T\right) \\
= & -k T \ln \mathscr{P}+N_{d} \mu_{o}+N_{u} \mu_{w} .
\end{aligned}
$$

In this equation, $p$ is the common pressure to which the microemulsion and its pure components are subjected, and the quantities $\mu_{o}$ and $\mu_{w}$ are the chemical potentials of the pure components, the same as in Eq. (1). The final form of $G_{\text {estimate }}$ follows immediately when we add the surface contribution,

$$
G_{\text {estimate }}=-k T \ln \mathscr{P}+N_{o} \mu_{o}+N_{u} \mu_{w}+\gamma \Sigma .
$$

The free energy $G_{\text {estimate }}$ is only an estimate of $G_{\text {exact }}$ since the surface free energy is not treated in detail, but is simply added afterwards as the term $\gamma \Sigma$. However, this estimate is physically consistent in that it is based on the partition function $Q$ that represents the sum over states in phase space. Thus the central requirement of Eq. (4) will be satisfied. The estimate is also consistent with the phenomenological model, as epitomized by Eq. (1), where the interfacial effects are summarized by the separate term $\gamma \Sigma$, and where the remaining free energy is handled as though it corresponded to that of a bulk system augmented by "mixing" effects.

Substitution of Eqs. (2) and (10) into Eq. (4) then yields

$$
\Omega(\lambda)=\Omega\left(N_{o}, N_{w}, \Sigma\right) \cong \mathscr{P}\left(N_{o}, N_{w}, \Sigma\right),
$$

where we use $\cong$ to indicate the mild approximations that have led to Eq. (10), approximations that do not change 
the order of magnitude of the result. Equation (11) also indicates that the physically consistent entropy of mixing is prescribed essentially by

$$
S_{\text {mix }}=k \ln \mathscr{P} \text {. }
$$

Now, $\Omega$ in Eq. (11) is computed by permuting cells that are filled with continuous fluids of oil or water while $\mathscr{P}$ in Eq. (11) is derived from the permutation of molecules, both types of permutation restricted by the exact same requirements. Under what conditions can these different types of permutations be nearly equal in number so that Eq. (11) will be satisfied? The answer is fairly simple. Think of each fluid molecule involved in the evaluation of $\mathscr{P}\left(N_{o}, N_{w}, \Sigma\right)$ as having been "smeared-out" throughout its imaginary cell in Fig. 3 . All cells will then be filled with imaginary continuous fluids, each with an average volume given by the average volume per molecule,

$$
\langle v\rangle=\rho^{-1},
$$

as discussed above. Now consider the phenomenological model. For simplicity, assume that we use a simple cubic lattice so that the cell volume will be

$$
v=\lambda^{3} \text {. }
$$

Since the phenomenological model cells and the imaginary cells (involving "smeared-out" molecules) are now both filled with continuous fluid, the permutations in both cases can be thought of as permutations of fluids among cells. The respective numbers of permutations will be almost equal if the numbers of cells are almost equal. This requires

$$
v \cong\langle v\rangle
$$

or, from Eqs. (13) and (14),

$$
\lambda \cong \rho^{-1 / 3} \text {. }
$$

Equation (11) assures that $k \ln \Omega$ is a physical entropy and will be satisfied by choosing the length scale prescribed by Eq. (16).

At this point, it should be re-emphasized that the length scale that forms the subject of this paper is specifically the scale for the configurational entropy, and is not that which measures either the curvature of the interface, the "granularity" of the microemulsion, or the persistence length.

\section{A SIMPLE EXAMPLE OF THE LENGTH SCALE $\lambda$}

In this section we present a concrete example of the general argument advanced above, but emphasize that the above estimate is not restricted by the conditions of this convenient, but simple, example. For this purpose we focus on a one-dimensional microemulsion of length $L$. The mesophases may now be referred to as globules, and $\Sigma$ is simply the number of interfaces between globules or, alternatively, the number of "ends" of the globules of one component. Thus, in one-dimension, the number of oil globules $=$ the number of water globules $=M=\Sigma / 2$.

First, we use the phenomenological model and calculate $\Omega$ by using a lattice having parameter $\lambda$. It follows immediately that the system will contain $C_{o}=\varphi L / \lambda$ oil cells, and $C_{w}=(1-\varphi) L / \lambda$ water cells. Then, it is easy to show ${ }^{11}$ that the number of distinct configurations (permutations of cells-random except for the constraint of fixed $\Sigma)$ is

$$
\Omega(\lambda)=\frac{\left(C_{o}-1\right) !}{\left(C_{o}-M\right) !(M-1) !} \frac{\left(C_{w}-1\right) !}{\left(C_{w}-M\right) !(M-1) !} .
$$

Now we evaluate $\mathscr{P}$ for the one-dimensional microemulsion as a system of $N_{o}$ oil molecules and $N_{w}$ water molecules confined to the length $L$, and limited to the same number of interfaces $\Sigma$. The number of allowed permutations of molecules is ${ }^{11}$

$$
\begin{aligned}
& \mathscr{P}\left(N_{o}, N_{w}, \Sigma\right) \\
& \quad=\frac{\left(N_{o}-1\right) !}{\left(N_{o}-\Sigma / 2\right) !(\Sigma / 2-1) !} \frac{\left(N_{w}-1\right) !}{\left(N_{w}-\Sigma / 2\right) !(\Sigma / 2-1) !} .
\end{aligned}
$$

Since $M=\Sigma / 2, \Omega(\lambda)$ in Eq. (17) and $\mathscr{P}\left(N_{o}, N_{w}, \Sigma\right)$ in Eq. (18) are of the same form, and Eq. (13) will be satisfied when $C_{o}=N_{o}$ and $C_{w}=N_{w}$, that is, when

$$
\lambda=\frac{L}{N_{o}+N_{w}}=\frac{L}{N}=\frac{1}{\rho},
$$

where $\rho$ is the linear number density. Thus, for the onedimensional case, the proper length scale is $1 / \rho$, or the length per particle in the fluid! This result, for the onedimensional case, is exact, since $\mathscr{P}\left(N_{o}, N_{w}, \Sigma\right)$ in Eq. (18) is exact, because, for the one-dimensional system, the specification of the "surface area" is unique, since it is equal to the total number of globules.

Some recent work by Sturgeon and Reiss, ${ }^{9}$ in which Eq. (4) was used, produced a length scale that displayed a factor whose size was on the order of $1 / \rho$ multiplied by an exponential factor that became dramatically small as $\langle n\rangle_{0}$ (the average number of molecules in an oil globule) increased. This factor can now be shown to be due to the omission of the second component in the microemulsion. Although the analysis of Sturgeon and Reiss was correct, the system they treated represented as assembly of superheated drops rather than a microemulsion.

Before leaving this section, we emphasize that onedimensional examples, though useful for certain illustrative purposes, for example to illustrate the qualitative properties of the mixing entropy, can only represent limited descriptions of microemulsions since they cannot incorporate bending energy, or exhibit phase transitions.

\section{THE ERROR IN THE RANDOM MIXING MODEL}

Having found the proper length scale, it is clear that the RMM overestimates it. We examine the implications of this fact by considering, again, a one-dimensional case. Before proceeding, it is useful to emphasize that we will be dealing with three different mixing entropies although two of them will be almost the same. First, there is the RMM mixing entropy, evaluated by the RMM method, and which we denote by $S_{\text {mix }}$ (RMM). Second, there is the entropy which, like $S_{\text {mix }}$ (RMM), is evaluated within the 
TABLE I. Values of the ratio: $S_{\text {mix }}(\mathrm{PM}) / S_{\operatorname{mix}}(\mathrm{RMM})$ for different values of $\langle n\rangle_{0}$ with $\varphi=0.5$.

\begin{tabular}{cccccc}
\hline \hline$\langle n\rangle_{0}$ & 10 & 50 & 100 & 500 & 1000 \\
\hline$S_{\operatorname{mix}}(\mathrm{PM}) / S_{\operatorname{mix}}(\mathrm{RMM})$ & 2.34 & 3.54 & 4.04 & 5.20 & 5.70 \\
\hline \hline
\end{tabular}

phenomenological model (PM), but using the correct scale. We denote this quantity by $S_{\text {mix }}(\mathrm{PM})$. Finally, there is the actual physical entropy, evaluated in a manner consistent with the phase space (PS) requirement and involving the permutation of molecules and prescribed by Eq. (12). This entropy we denote by $S_{\operatorname{mix}}$ (PS).

From the previous discussion we know that

$$
\begin{aligned}
& S_{\mathrm{mix}}(\mathrm{RMM})=k \ln \Omega(\xi), \\
& S_{\mathrm{mix}}(\mathrm{PM})=k \ln \Omega(\lambda), \\
& S_{\mathrm{mix}}(\mathrm{PS})=k \ln \mathscr{P} .
\end{aligned}
$$

Since $\lambda$ is to be chosen so that $\Omega(\lambda) \cong \mathscr{P}$, it follows that

$$
S_{\text {mix }}(\mathrm{PM}) \cong S_{\text {mix }}(\mathrm{PS}) \text {. }
$$

However, $S_{\text {mix }}$ (RMM) may have a very different value, and it is this difference that we now study. Furthermore, since we want to focus on the correct use of the phenomenological model, it is $S_{\text {mix }}$ (PM) rather than $S_{\text {mix }}$ (PS) that should be compared with $S_{\text {mix }}$ (RMM), even though in accordance with Eq. (23), both entropies have almost the same value.

We already know that the correct $\Omega(\lambda)$ is given by Eq. (17) with $C_{o}, C_{w}$, and $M$ set equal to $N_{o}, N_{w}$, and $\Sigma / 2$, respectively, i.e., by the right-hand side of Eq. (18). Furthermore, under these conditions $\lambda$ is given by Eq. (19). Substituting this value of $\lambda$ into Eq. (21), as well as $N_{o}$ $=N \varphi=M\langle n\rangle_{o}$, where $\langle n\rangle_{o}$ is the average number of oil molecules per oil globule, and $N_{w}=N(1-\varphi)=M\langle n\rangle_{w}$, where $\langle n\rangle_{w}$ is the average number of water molecules per water globule, we find, using Stirling's approximation,

$$
\begin{aligned}
S_{\text {mix }}(\mathrm{PM})= & k M\left[\langle n\rangle_{o} \ln \langle n\rangle_{o}+\langle n\rangle_{w} \ln \langle n\rangle_{w}-\left(\langle n\rangle_{o}-1\right)\right. \\
& \left.\times \ln \left(\langle n\rangle_{o}-1\right)-\left(\langle n\rangle_{w}-1\right) \ln \left(\langle n\rangle_{w}-1\right)\right] \\
= & -N k\left\{\varphi\left(1-\frac{1}{\langle n\rangle_{o}}\right) \ln \left(1-\frac{1}{\langle n\rangle_{o}}\right)+(1-\varphi)\right. \\
& \times\left[1-\frac{\varphi}{(1-\varphi)\langle n\rangle_{o}}\right] \ln \left[1-\frac{\varphi}{(1-\varphi)\langle n\rangle_{o}}\right] \\
& \left.+\frac{\varphi}{\langle n\rangle_{o}} \ln \left[1-\frac{\varphi}{(1-\varphi)\langle n\rangle_{o}^{2}}\right]\right\} .
\end{aligned}
$$

In the RMM the number of permutations of oil and water cells is

$$
\Omega(\xi)=\frac{C !}{C_{0} ! C_{w} !}=\frac{\left[\frac{M}{\varphi(1-\varphi)}\right] !}{\left(\frac{M}{1-\varphi}\right) !\left(\frac{M}{\varphi}\right) !}
$$

where $C=L / \xi, C_{o}=\varphi L / \xi$, and $C_{w}=(1-\varphi) L / \xi$ are the total number of cells, the number of oil cells, and the number of water cells, respectively, and where we have used

$$
M=C \varphi(1-\varphi)=\frac{L}{\xi} \varphi(1-\varphi)
$$

Note that Eq. (26) is the one-dimensional equivalent of Eq. (3). Introduction of Eq. (25) into Eq. (20), again using Stirling's approximation, leads to the following expression for $S_{\text {mix }}$ (RMM),

$$
S_{\text {mix }}(\mathrm{RMM})=-k \frac{N \varphi}{\langle n\rangle_{o}}\left[\frac{\ln \varphi}{1-\varphi}+\frac{\ln (1-\varphi)}{\varphi}\right] .
$$

Table I lists values of the ratio $S_{\operatorname{mix}}(\mathrm{PM}) / S_{\text {mix }}$ (RMM) for different values of $\langle n\rangle_{\circ}$ with $\varphi=0.5$. Since $\langle n\rangle_{o}$ in one dimension is usually of the order of $100,{ }^{11}$ we see that the ratio of the values of $S_{\text {mix }}$ is of the order of 4 . In three dimensions we might expect the ratio to be of the order of 12 . Thus, there is a large difference between the entropy evaluated by means of the RMM and that using the PM with the correct scale. The RMM fails to count a very large number of configurations.

Figure 4 also shows the difference between $S_{\text {mix }}$ (PM) and $S_{\text {mix }}$ (RMM). In this case $\varphi$ is varied while keeping $N=N_{o}+N_{w}$ and $M$ constant such that $N / M=200$. Note that because $M$ is constant, the thermodynamic states in Fig. 1 lie on a horizontal line in a Gibbs triangle. The values for the entropy of mixing for the two cases are significantly different, as they are in Table I, but the striking feature is that the entropies also behave qualitatively differently. While $S_{\text {mix }}$ (RMM) is minimum for the equi-

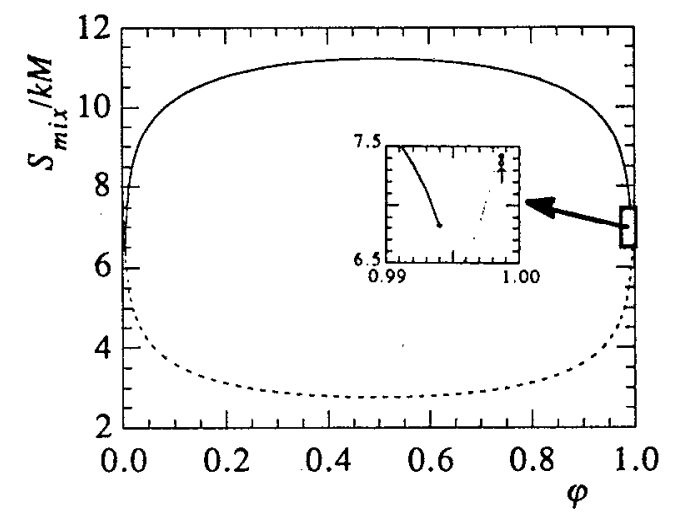

FIG. 4. Plots of $S_{\operatorname{mix}}$ (PS) and $S_{\operatorname{mix}}$ (RMM) (upper and lower curves, respectively) vs oil fraction $\varphi$, for fixed interfacial area $\Sigma$. Inset illustrates the lower limit of $S_{\text {mix }}$ if the upper curve is considered to represent $S_{\operatorname{mix}}(\mathrm{PS})$. 
molecular solution $(\varphi=0.5)$ and increases as $\varphi$ tends to 0 or $1, S_{\text {mix }}$ (PM) exhibits the opposite behavior.

The thermodynamic consequences of this disparate behavior of the mixing entropies is best considered by referring to Eq. (1), and, especially to the last two terms, $G_{\text {mix }}$ $=\gamma \Sigma-T S_{\text {mix }}$ in that equation. Assume that at $\varphi=0.5$, $G_{\text {mix }}(\mathrm{RMM})$ and $G_{\text {mix }}(\mathrm{PM})$ are of about the same magnitude [perhaps because in $G_{\text {mix }}$ (RMM) a value of $\gamma$ somewhat higher than the actual value had been used-a distinct possibility since $\gamma$ is known to be very small and its value is not known with absolute accuracy]. Then as $\varphi$ is varied to either the oil or water rich compositions, $G_{\text {mix }}$ (RMM) will decrease while $G_{\text {mix }}(\mathrm{PM})$ will increase, thus stabilizing and destabilizing the system, respectively. However, other factors such as bending energy act to destabilize the system and the range of stability is limited to intermediate values of $\varphi$. On the other hand, in the correct treatment, both entropy and bending energy act in the same direction so that the predicted range of stability is narrower. Thus the error implicit in the random mixing approach leads to the prediction of a range of stability that is too broad. Such concerns involving the entropy of mixing in both oil rich and water rich domains have been voiced qualitatively by Gompper and Schick. ${ }^{12}$

Figure 4 shows that the values of $S_{\text {mix }}$ (RMM) and $S_{\text {mix }}(\mathrm{PM})$ are very close in the oil and water rich domains. It is apparent from Eq. (26) that this must be the case, since the length scale in the RMM becomes of molecular size (i.e., close to the exact length scale) when $\varphi \rightarrow 0$ or $\varphi \rightarrow 1$. For most values of $N / M$, it can be shown that $S_{\text {mix }}$ (RMM) and $S_{\text {mix }}$ (PM) are equal at two values of $\varphi$ (symmetric with respect to $\varphi=0.5$ ). The physical reason for this behavior is the following. For any choice of $\xi$, the random mixing of oil and water cells leads to a particular average or most probable extent of interface $\Sigma$ given by Eq. (26). If the amount of surfactant were such as to require this value of $\Sigma$ for a saturated layer, then the surfactant would not be exercising any constraint on the system. Now suppose that both $\xi$ and $\Sigma$ were fixed arbitrarily, e.g., suppose that $\xi=\lambda=1 / \rho$ so that $\xi$ represented the correct length scale. Then, the only way in which Eq. (26) could be satisfied would be to have $\varphi$ in the equation set to a value to maintain the equality. Equation (27) then becomes a quadratic equation in $\varphi$ whose roots specify the two compositions at which the RMM gives (by accident) the correct result.

A few remarks concerning $S_{\text {mix }}$ (PS) are appropriate. As we have indicated, this quantity is essentially represented by the upper curve in Fig. 4. However, since in the evaluation of $S_{\text {mix }}$ (PS) the system is viewed as consisting of discrete molcculcs rather than as cells occupied by continuous fluid, $\langle n\rangle_{o}$ and $\langle n\rangle_{w}$ cannot be less than unity. This means that the curve for $S_{\text {mix }}$ (PS) must terminate at sufficiently oil or water rich compositions. Such termination is illustrated by the magnified inset in Fig. 4. In the inset the solid curve shows the decreasing $S_{\text {mix }}$ (PS) while the dotted curve shows $S_{\text {mix }}$ (RMM). The latter continues upwards to the absurd result of an infinite value for the mixing entropy in a single component system where there can be no such entropy, but the former terminates at a lower limit and the curves never cross.

The departure from a relatively constant length scale has serious implications. According to Eqs. (1) and (2), $\Delta G_{i j}$, the Gibbs free energy difference between two macroscopic thermodynamic states $i$ and $j$ of the system will contain the term $\Delta S_{\mathrm{mix}, i j} \equiv S_{\mathrm{mix}, j}-S_{\mathrm{mix}, i}=k \ln \left(\Omega_{j} / \Omega_{i}\right)$. Because only the ratio $\left(\Omega_{j} / \Omega_{i}\right)$ appears, the length scale will cancel out of $\Delta S_{\text {mix }, i j}$ if it is constant and independent of the thermodynamic state. Thus knowledge of its magnitude will be unnecessary. The proper length scale is almost independent of thermodynamic state whereas, the scale introduced in the RMM is very dependent, since it is given by Eq. (26).

It might be argued that even if the length scale were not constant, and depended on thermodynamic state, no great harm would result as long as the variation of scale added nothing to the free energy of the microemulsion phase beyond terms linear in $\varphi$ and/or $(1-\varphi)$. Comparison of Eqs. (24) and (27) [note that $\varphi\langle n\rangle_{o}=(1-\varphi)\langle n\rangle_{w}$ ] shows that is not the case.

It might also be argued that the variation in $S_{\text {mix }} / k M$ as a function of $\varphi$ far from the edges of the diagram in Fig. 4 is small enough to be unimportant with regard to the stability of the microemulsion. On the other hand, this variation must be balanced against a surface free energy per surface molecule which is also very small, so that the variation in $S_{\text {mix }} / k M$ remains relatively important in determining stability. Note that in one dimension, $2 M$ is of the order of the number of surface molecules so that $S_{\mathrm{mix}} /$ $k M$ is of the order of the mixing entropy per surface molecule.

\section{CONCLUSION AND ASSESSMENT}

There exists a large and successful community vigorously addressing the subject of microemulsions. Sophisticated theory has been advanced for the interpretation of experimental results. The concepts of bending energy, persistence length, and related ideas have added much to the field, but mixing entropy remains a central issue in characterizing the stability behavior of the microemulsion phase. ${ }^{13}$

It is therefore remarkable that (apparently), with the exception of Ref. 9, previous authors have not subjected their definitions of mixing entropy to the test of physical consistency. Instead, the mixing entropy, introduced in an intuitive manner, becomes the subject of intense mathematical manipulation. Sometimes the intuition is very good. For example, in the case of the strict lattice model, ${ }^{14,15}$ as opposed to the phenomenological lattice, when the lattice parameter is chosen approximately equal to $\rho^{-1 / 3}$, the associated entropy of mixing will very nearly meet the test of physical consistency. In contrast, in the case of the RMM, the associated mixing entropy fails seriously to meet this test except at the unique compositions indicated in Sec. III. Of course, this is not to depreciate the outstanding work in the field, but only to call attention to the fact that, due to the large values of the length scale used, the RMM accounts for only part of the actual entropy of mixing. Only 
through the use of the fundamental length scale for configurational entropy, estimated in Sec. III, can the proper entropy of mixing be evaluated.

How does the problem of physical consistency with $S_{\operatorname{mix}}$ arise? As Eq. (3) implies, $k \ln \Omega$ is a (configurational) entropy, and $\Omega$ is a number of "states" in configuration space. However, the proper way to evaluate physical entropy is through the counting of quantum states in phase space. In the semiclassical phase integral ${ }^{11}$ the de Broglie wavelength $\Lambda$ is the natural scale of length that is used to transform the configuration integral into a pure number, specifically, into the partition function. When configurational entropy, in this case mixing entropy, is evaluated in configuration space as opposed to a more fundamental evaluation in phase space, there is a need for a length scale, and the physical consistency of the entropy of mixing is not assured unless the choice of this length scale is consistent with the fundamental definition involving phase space. The problem is compounded by the fact that configurational entropy cannot be extracted from the total entropy in any absolute manner. It must always be defined and then usually within the confines of a particular model.

Phenomenological models of microemulsions that use "large" length scales, such as the RMM, seriously underestimate the entropy of mixing. Where does this omitted entropy come from? When a cell is permuted on a lattice, say for example, when a cell is moved to the adjacent cell on the right, it must be translated a distance $\lambda$, exactly. Yet, a continuum of intermediate positions are possible in the real system, but they are not counted. Each and every one of these possible positions should contribute to the configurational entropy, but do not.

In the core derivation in Sec. III, physical consistency is assured through the satisfaction of Eq. (4). The use of $G_{\text {exact }}$ assures that the enumeration of configurations in configuration space is consistent with the enumeration of quantum states in phase space. Failure to meet this test can reduce the defined entropy of mixing to a counting of fuzzily defined patterns of the microemulsion so that it becomes an information theoretic rather than a physical entropy. Again, it is to be emphasized that the strict test of consistency has, to our knowledge, not been applied, except in Ref. 9.
It may be argued that the conventional approaches have nevertheless yielded results that are reasonably consistent with experiment. Even though this may be the case, the problem cannot be ignored, since without the test of consistency there is actually no physics in the theory of mixing entropy! Features such as bending energy and persistence length do of course contain physics, but the mixing entropy is usually fundamental to the entire theory. In fact, while bending energy is a well-defined physical concept, for example in the physics of membranes, its use in some phenomenological theories of microemulsions, for example the RMM, may actually obscure an incorrect evaluation of the mixing entropy. Figure 4 hints at this since the decline of the correct entropy, in the oil and water rich emulsions, has the same qualitative effect as bending energy.

\section{ACKNOWLEDGMENTS}

This work was supported by the National Science Foundation under NSF Grant No. 4-443835-HR-21116. J.A.M. wishes to thank the Spanish Ministry of Education and Science for financial support through a grant of the Fulbright Program.

'P. A. Winsor, Trans. Faraday Soc. 44, 376 (1948).

${ }^{2}$ H. Reiss, J. Colloid Interface Sci. 53, 61 (1975).

${ }^{3}$ Y. Talmon and S. Prager, J. Chem. Phys. 69, 2984 (1978).

${ }^{4}$ E. Ruckenstein and J. Chi, J. Chem. Soc. Faraday Trans. 2 71, 1690 (1975).

${ }^{5}$ P. G. De Gennes and C. Taupin, J. Phys. Chem. 86, 2294 (1982).

${ }^{6}$ J. Jouffroy, P. Levinson, and P. G. De Gennes, J. Phys. (Paris) 43, 1241 (1982).

${ }^{7}$ B. Widom, J. Chem. Phys. 81, 1030 (1984).

${ }^{8}$ D. Andelman, M. E. Cates, D. Roux, and S. A. Safron, J. Chem. Phys. 87, 7229 (1987).

${ }^{9} \mathrm{~K}$. Sturgeon and H. Reiss, J. Chem. Phys. 98, 1493 (1993).

${ }^{10}$ M. Kahlweit and H. Reiss, Langmuir 7, 2928 (1991).

${ }^{11}$ T. L. Hill, Introduction to Statistical Thermodynamics (AddisonWesley, Reading, 1960).

${ }^{12} \mathrm{G}$. Gompper and M. Schick, in Micelles, Membranes, Microemulsions, and Monolayers, edited by W. M. Gelbart, A. Ben-Shaul, and D. Roux (Springer, New York, 1993), Chap. 8.

${ }^{13}$ L. Golubović and T. C. Lubensky, Phys. Rev. A 41, 4343 (1990).

${ }^{14}$ B. Widom, J. Chem. Phys. 84, 6943 (1986).

${ }^{15}$ K. A. Dawson, B. L. Walker, and A. Berera, Physica A 165, 320 (1990). 